\title{
Application of Ergonomics Theory Based on Color Perception Theory in Business Design
}

\author{
Chen $\mathrm{Ni}^{1}$, Li Wang ${ }^{1}$ \\ ${ }^{1}$ School of Design\&Art, Dalian Institute of Science and Technology, Dalian, LiaoNing, 116052, China
}

\begin{abstract}
There is an obvious difference between the real world and the world we perceive, and we are constantly updating the definition of color. Color, like point, line, and plane, is the basic unit of our visual information. However, compared with other visual elements, our research on color has obvious shortcomings. We can solve many specific problems in our design practice through new analysis methods.
\end{abstract}

\section{Human Visual System and Color Perception Theory}

\subsection{Human Visual System Theory}

Generally speaking, the nature of visual impressions is unlike linguistics, which has rigorous language logic. As soon as human beings are born, they have the ability of visual perception. Different from linguistics, which requires a process of communicating and learning with people, newborn babies can discern things around a few weeks after birth, but it takes one to two years to master the most common everyday language.[1] General research believes that the transmission and understanding of visual information need not be as accurate as learning languages, but the limitations of our understanding of human visual perception systems have led us to ignore the role of complex underlying information and unique connotations in visual language.

An Indian medical scientist, Pawan Sinha, helped a large number of children with congenital eye diseases regain their sights in his Prakash project. And corresponding tests are performed on the recovery of the visual cognitive system of these children every week. This follow-up survey will last forty weeks or more from the time these children recover vision after receiving treatment. This survey provides valuable information for understanding how human visual system is constructed. Unlike newborn babies, these children have mastered the language system before the visual system is built, so they can describe the visual signals they capture in language. The research of The Prakash project includes the causal link between the visual information that humans come into contact with early and the visual experience formed later. It turns out that humans learn visual knowledge in a similar way to language learning. However, what has

E-mail: nichen93@hotmail.com important reference value to this paper is Singha's research on how the human visual system interprets images into objects. Our visual systems only receive color signals with blocky shapes or different gloss changes, which are then summarized by our brain as objects with abstract meaning or objective objects after making complex judgments. In other words, the world we see is the result of subjective processing of the received color information by our brain.

\subsection{Subjective Color Perception Theory}

There is an obvious difference between the real world and the world we perceive. This difference is attributed to illusions by us, which means that illusions capture the essence of the work of our sensory system, just as magic shows are performances that use the illusions of our perception systems. We experience illusions every day in our daily lives. There are many times when the conclusions reached by our visual organs are inconsistent with the conclusions reached by our perceptual system. Our sensory system is always influencing our senses, whether it is sight, hearing or touch, so many of the images we "see" are nothing more than fictional worlds in our heads. The ever-changing nature requires humans to respond quickly and accurately to visual information (research shows that this process takes only 150 milliseconds). In order to complete this process quickly, human instincts evolved over millions of years have made our brains predict scenarios all the time, so many times what we see is not what is happening but what is going to happen. There is an old saying in China that "seeing is believing", from which it can be seen that visual information remains the way we rely on most to perceive the world during millions of years of evolution. However, such a main form of obtaining information is still highly subjective, that is, the world we see is still very different from the real world. The illusion effect 
mentioned earlier is often used in the creation of many works of art. It aims to confuse the world of human consciousness with the world of objective existence. Modern medical research shows that the visual information transmitted to the back of the brain through the ganglion cerebral only accounts for $10 \%$ of the total information, and the remaining $90 \%$ comes from other parts of the brain. Many people attribute the cause of illusion to the fragility of human consciousness, but this is not the case. The evolution of human sensory system is not towards the more and more real world that can be seen. In fact, the definition of the "real" world in our consciousness changes as the surrounding environment changes.[2] As for color, we will continue to update its normal definition. In the future art creation or design activities, creation based on the plasticity of the brain will be a new direction for the development of art and design.

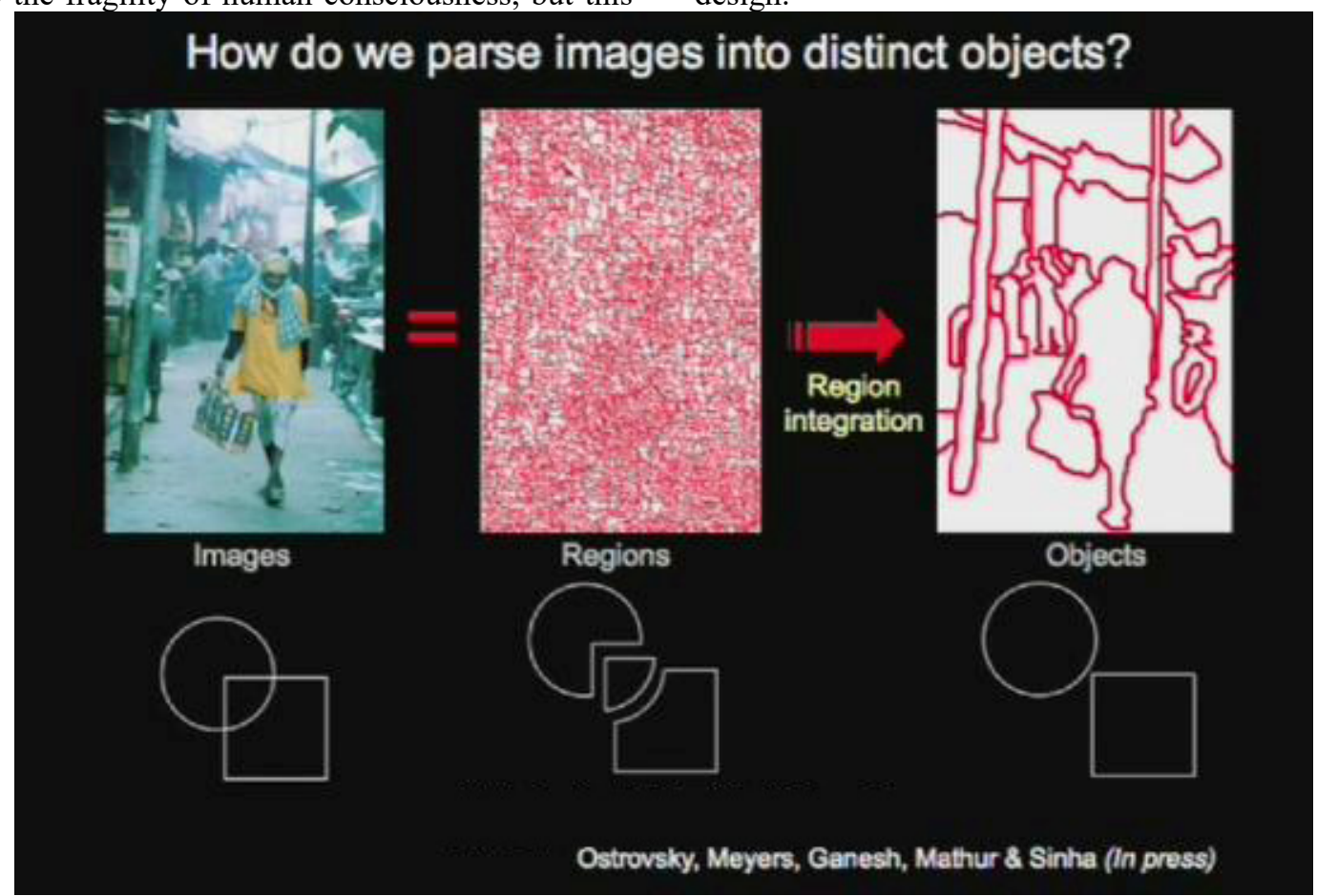

Figure 1 . The theory of we parse images into distinct object.

\section{Ergonomics Analysis Based on Color Perception Theory}

\subsection{Biological Dominance}

People's perception of color has both commonalities and differences, which means that the effect of color on people is different. Some color symbols or matching are common throughout the world, such as yellow and black stripes used to warn of danger. That is to say, the color contrast relationship formed by this type of color power is derived from biological instincts. Colors based on this effect can be called biologically dominant colors. Perhaps not just for humans, this type of color effect is equally effective for some animals. Someone has investigated children's preferences for color. After comparison, it can be found that red and yellow are popular colors for children all over the world. Children have not been disturbed too much by the outside world, so their preference for color is closer to instinct. As people age, the leading role of region and environment will gradually be strengthened.[3] Thus, in the practice of art design involving younger target groups, we need to choose and consider the color relationship from the perspective of biological dominance.

\subsection{Environmental Dominance}

In many cases, the image of color in people's minds reflects the difference. For example, surveys show that people near the equator generally like to use the matching of warm colors, while in the middle and high latitudes, people are more inclined to green and blue colors. In other words, people's perception of color will be affected by regions, and we can call it regional dominant color. Under normal circumstances, even the same color will bring people different psychological feelings in different environments. One type of color will be welcome in this area, but it is likely to be rejected in another. Of course, this is not just caused by geography. The factors that control people's preferences are all extremely complicated, so sometimes we should adopt a more tolerant attitude to color issues.

\subsection{Humanistic Dominance}

Here is another case: in China, yellow is a symbol of royalty, a noble symbol that ordinary people cannot reach. however, according to legend, Judas wears yellow clothes, 
so in Christian countries, yellow has shameful, betrayal and despicable meaning. Investigating the reason, we can attribute this kind of color matching difference to humanistic aspect, and we can also call it humanistic dominant color.

The above cases indicate that the contrast effect of color or the image of color in different people's consciousness are quite different. However, this does not mean that there are limitations of color forces and contrast relationship between colors. We have always emphasized that human vision is very subjective. The contrast effect between certain colors will be subjectively enhanced or weakened along with geographical, environmental or human influence. The above three forms are the main forms of ergonomic application through color perception theory.

\section{Conclusion}

Color, like point, line, and plane, is the basic unit of our visual information. However, compared to other visual elements, our research on color has obvious shortcomings. We can use the above analysis methods to solve many specific problems in our design practice.

For example, the rise of older consumers. After 2025, global aging will reach a new stage. After 2025, the frequency of using fonts under 13 points will be greatly reduced, because most middle-aged and elderly people cannot see those words clearly. Similarly, use yellow with caution. This is an example of biological dominance. After the age of 50, the amount of light that enters our retina is much lower than when we are young, and the colors lost first are bright yellow and light yellow. So many things passed through those bright yellow fonts are not visible to a large part of people. Therefore, we should use black, white and red to convey important information Writing in black, white and red with strong contrast is more suitable for the world of the elderly. We should consider the majority when doing commercial design, because in the future, most of the people targeted for design services will be elderly people. Many people retire at the age of 50-60. As people become more and more long-lived, it is normal to live to the age of 100 . So now people spend more than half of their lives in old age. When designing projects, we need to think more about the elderly. [4]

Modern psychological research shows that color not only brings warm and cold sensory effects to people, but also affects how we feel about distance. Studies abroad have shown that the highest accident rate among cars of various colors is blue Color cars, followed by green, gray, white, red, and black. Studies abroad show that among cars of various colors, the cars with the highest accident rates are blue cars, followed by green, gray, white, red, and black. Different colors produce different effects on our consciousness. Among them, the colors that appear convex are called advancing colors, and the colors that appear concave are receding colours.

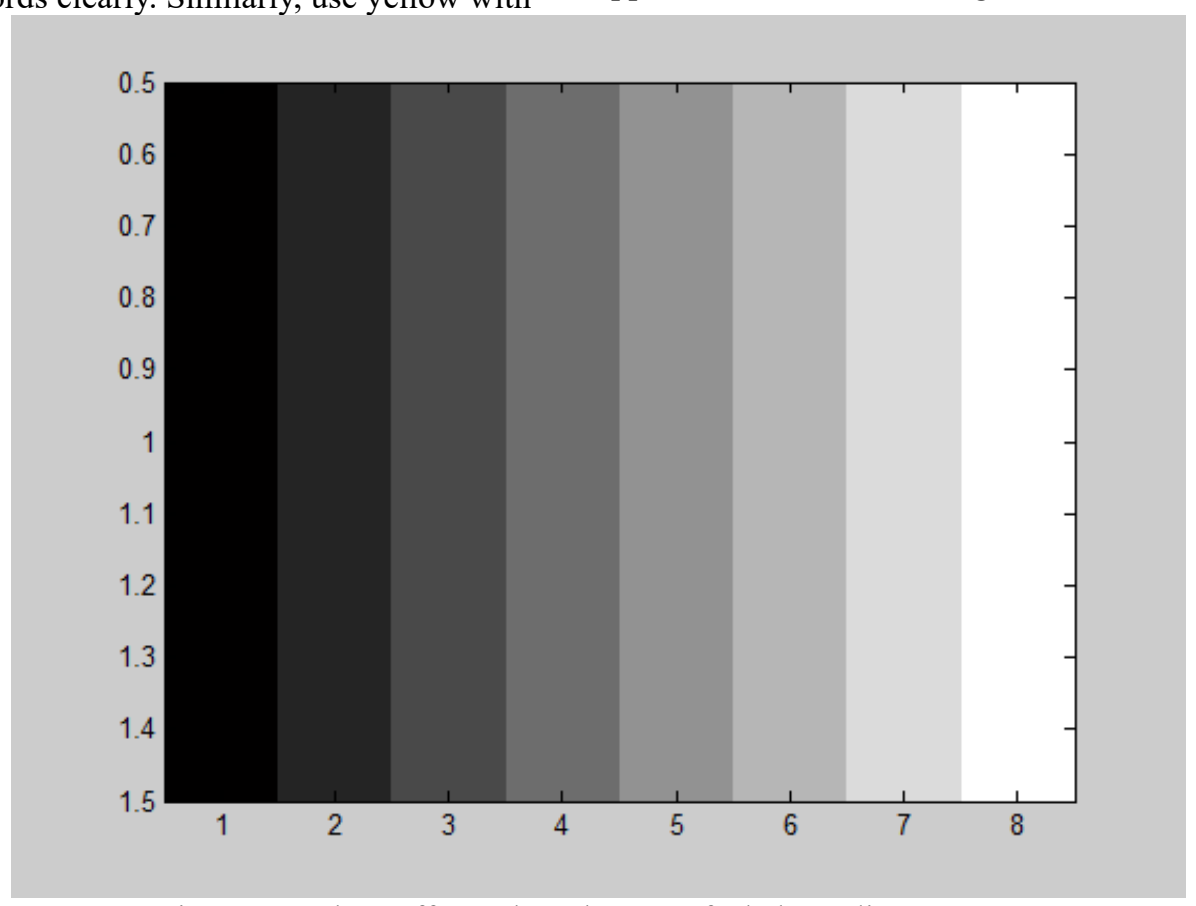

Figure 2. Colour affects about how we feel about distance.

Blue is the color that gives us the strongest receding sense, so blue cars look farther than they actually are. There are various reasons for a car accident, so it is impossible to simply associate the car color with the cause of a traffic accident. In addition, different time periods, different lighting conditions, and different angles have different visual effects on car colors. However, the correlation between color properties like the visibility of car color, advancing color, receding color and different accident rates is obvious. [5] Similar cases are very common in ergonomic design. If we continue to study in depth from this perspective, the research will provide clearer guidance for the application of color in design in the future.

\section{References}


1. Zhang haichao (2015) A Discusison on the Level of Requirements and Design Strategies in Interactive Design.Art Science and Technology(11)288

2. Corney, D, Haynes, J, Rees, G, \& Lotto, RB.(2009)The Brightness of Colour. PLoS ONE.New York

3. Hulme, D.J., Hirsch, R., Buxton, B.F. and Lotto, R.B. (2007)A new reduction from 3SAT to n-partite graphs. Refereed Proceedings: IEEE.L.A.

4. Deng Yanmin (2019) A Preliminary Analysis of Humanized Design of Product Design. Art Science and Technology(4)284

5. Zhuang fei (2013) Ergonomic in the Design of Industrial Products.Journal of Jiamusi Education Institute.(8)186 